$\begin{array}{llll}\text { Submission: 14/02/2019; } & 1^{\text {st }} \text { round notif.: 21/05/2019; } & \text { New version: 04/07/2019; } & 2^{\text {nd }} \text { round notif.: 13/08/2019 } \\ \text { Camera ready: 14/01/2020; } & \text { Edition review: 21/02/2020; } & \text { Available online: 31/05/2020; } & \text { Published: 31/05/2020 }\end{array}$

\title{
Utilização de Enriquecimento Semântico para a Recomendação Automática de Videoaulas no Moodle
}

\section{Use of semantic enrichment for the Automatic Recommendation of video lectures in Moodle}

Eduardo Barrére

Universidade Federal de Juiz de Fora

eduardo.barrere@ice.ufjf.br

Miguel Alvim de Almeida

Universidade Federal de Fora

miguel.alvim@ice.ufjf.br

\author{
Marluce Aparecida Vitor \\ Universidade Federal de Fora \\ marlucevitor@ice.ufjf.br
}

Jairo Francisco de Souza

Universidade Federal de Juiz de Fora

jairo.souza@ice.ufjf.br

\section{Resumo}

Considerando que os vídeos são mídias altamente atrativas aos alunos e que é possível agregar diversas informações que identifique-no de forma satisfatória, foi desenvolvida uma solução para realizar a recomendação automática de vídeos educacionais para o professor, que por sua vez pode disponibilizá-los aos alunos de uma determinada turma, inicialmente na plataforma Moodle. A solução usa como fonte um vídeo previamente processado, de forma a obter os principais termos (sistema de indexação) presentes no áudio do vídeo e o relacionamento (recomendação) desse vídeo com os demais do repositório. Com base nos termos de busca, o sistema recomenda um conjunto de vídeos e o professor seleciona os que pretende divulgar para a turma. Foi realizado um experimento que comprovou a corretude e viabilidade de uso para a recomendação de vídeos educacionais na plataforma Moodle.

Palavras-Chave: Recomendação Automática; Sistema de Indexação; Vídeos Educacionais

\begin{abstract}
Considering that videos are highly attractive to students and that it is possible to aggregate several information that identifies a video in a satisfactory way, a solution has been developed to carry out the automatic recommendation of educational videos to the teacher, who in turn can make them available to the students of a certain class, initially in the platform Moodle.The solution uses as a source a video previously processed, in order to obtain the main terms (indexing system) present in the audio of the video and the relationship (recommendation) of that video with the other of the repository. An experiment was carried out that confirmed the correctness and feasibility of use for the recommendation of educational videos in the Moodle platform.
\end{abstract}

Keywords: Automatic Recommendation; Indexing System; Video Lectures

Cite as: Barrére, E., Vitor, M. A. Almeida, M. A. de \& Souza, J. F. de. (2020). Use of semantic enrichment for the Automatic Recommendation of video lectures in Moodle (Utilização de Enriquecimento Semântico para a RecomendaçãoAutomática de Videoaulas no Moodle). Brazilian Journal of Computers in Education (Revista Brasileira de Informática na Educação - RBIE), 28, 319-334. DOI: 10.5753/RBIE.2020.28.0.319 


\section{Introdução}

Na última década, a educação a distância (EaD) apresenta um crescimento significativo, especialmente devido à maior acessibilidade, por parte da população, às tecnologias de acesso digital e à internet (Allen \& Seaman, 2017), se tornando, de fato, o principal meio de acesso ao ensino para muitas pessoas e até para divulgação científica (Tibaná-Herrera \& Moya-Anegón, 2018).

Uma das principais plataformas para viabilizar a EaD é o Moodle (Modular ObjectOriented Dynamic Learning Environment). Neste mesmo cenário, existe a tendência ao uso de vídeos educacionais, também chamados de videoaulas. É possível considerar o vídeo como a mídia da nova geração (Pirhonen \& Rasi, 2016) (Brame \& Perez, 2016), pois ele pode ser gerado com recursos tecnológicos disponíveis em grande escala (smartphones, notebooks etc), é de fácil produção e apresenta grande atratividade cognitiva (imagem e som ao mesmo tempo). Um ponto fundamental para a popularização dos vídeos são os repositórios de armazenamento e compartilhamento, com destaque para o YouTube, que, além de armazenar vídeos de conteúdo geral, também é utilizado por alguns de seus usuários para disponibilizar vídeos educacionais. Outros repositórios, como Video@RNP, NTNU e VideoLectures.NET, também são utilizados para fins educacionais.

Apesar de ser a plataforma mais utilizada para o ensino a distância (Damnjanovic \& Mijatovic, 2015), o Moodle nem sempre acompanha o surgimento de novas tecnologias e métodos para a EaD. Esse problema é, em geral, sanado com a criação de plugins, que adicionam e/ou expandem as atividades existentes na ferramenta. Nesse contexto, foram incorporadas ao Moodle soluções para gamificação (Barrére et al., 2017), para recomendação de objetos de aprendizagem (Nascimento et al., 2017), entre outras.

No caso específico do Moodle, as versões recentes já permitem o acesso aos repositórios de vídeos de forma integrada. Mas tal integração ainda é um ponto a ser explorado, pois aspectos como recomendação e colaboração ainda necessitam de maior investigação. Com vista a esse problema, este trabalho apresenta um plugin para o Moodle, com o intuito de incorporar aspectos de pesquisa e recomendação de vídeos educacionais oriundos de um repositório. Esta solução utiliza como repositório de vídeos o serviço VideoAula@RNP da Rede Nacional de Ensino e Pesquisa (RNP), acessado pelo sistema BAVi (Busca Avançada de Vídeo), que realiza o processamento da base de vídeos do VideoAula@RNP, ampliando os termos de busca associados a cada vídeo e também uma relação de similaridade entre os vídeos da base. Dessa forma, buscou-se desenvolver um sistema de recomendação automática em um ambiente real e com um número significativo de potenciais usuários.

O artigo está organizado de forma a, primeiramente, apresentar características importantes sobre sistemas de recomendação e de repositórios de mídias. Em seguida, apresenta o sistema BAVi e a solução proposta e por último, é apresentada uma prova de conceito da aplicação prática do plugin proposto.

\section{Sistema de Recomendação}

Segundo Costa et al. (2013), é desafiador para os professores selecionar e organizar os diversos recursos educacionais que vão surgindo na $W e b$, de forma a contribuir para o aumento do potencial de aprendizagem e da motivação dos alunos. Devido ao volume de recursos existentes, surge a dificuldade de filtragem de conteúdo específico sobre certo tema. 
O desenvolvimento de trabalhos focados na criação de sistemas de recomendação para plataformas de aprendizagem não é algo novo, como pode ser visto em (Ribeiro et al., 2013), no qual apresenta um sistema para recomendação de objetos de aprendizagem baseado na análise de conteúdos postados nos fóruns de discussão em um curso do Moodle. Mas também não foi plenamente resolvido, apesar de avanços significativos, como os apresentados em:

- (Brandão et al., 2017): apresenta uma metodologia que emprega ontologias, anotação automática de metadados, recuperação de informação e mineração de texto para recomendar e agregar conteúdos relacionados, usando a categoria de metadados de relação definida pelo SCORM.

- (Limongelli \& Temperini, 2016): apresenta um sistema de recomendação para criação automática de cursos por parte dos professores com base numa base de cursos préarmazenada.

- (Halawa et al., 2015): Apresenta um modelo personalizado de recomendação de $e$-learning baseado no tipo psicológico e modelos de estilo de aprendizagem pré-definidos.

Mas quando o assunto é quais os plugins que viabilizam a recomendação na plataforma Moodle $^{1}$, o esforço gerado nas pesquisas não se reflete em recursos disponíveis para a comunidade, pois os três plugins de recomendação disponíveis não apresentam relação direta com os trabalhos acadêmicos. São eles:

1) Recommender $^{2}$ : tem como objetivo, recomendar atividades mais populares, cursos e bookmarks compartilhados. O plugin é dividido em três serviços: atividades populares, cursos populares e marcadores compartilhados pelo professor;

2) Analytics and Recommendations ${ }^{3}$ : é uma ferramenta para estudantes e professores. Ele mostra de maneira visual a participação dos alunos em cada atividade do curso, assim como recomendações para os alunos sobre quais atividades devem trabalhar para melhorar sua nota final;

3) Recommendation letters and appraisals ${ }^{4}$ : permite que o participante solicite recomendações ou avaliações. A recomendação pode ser feita para qualquer pessoa, basta a mesma receber um link para preencher um formulário. O papel do professor/tutor é o de configurar perguntas, visualizar e aceitar respostas. Os formulários podem ser configurados quanto ao número máximo de pedidos e o número mínimo de recomendações completas para a conclusão da atividade.

O uso de plugins não é a única forma de agregar recomendação ao Moodle, pois ela pode ocorrer através de integração entre sistemas. Em (Ferreira \& Raabe, 2010), é apresentada uma abordagem para recomendação de objetos de aprendizado no Moodle. A abordagem utiliza como fonte de dados os metadados presentes no formato SCORM. Para cada acesso de mais de 20 segundos a um objeto de aprendizado, o sistema registra essa leitura em uma tabela do

\footnotetext{
${ }^{1}$ Portal de plugins do Moodle: https://moodle.org/plugins/

${ }^{2}$ Plugin Recommender: https://moodle.org/plugins/block_recommender

${ }^{3}$ Plugin Analytics and Recommendations: https://moodle.org/plugins/block_analytics_recommendations

${ }^{4}$ Plugin Recommendation letters and appraisals: https://moodle.org/plugins/mod_recommend
} 
usuário e, utilizando técnicas de filtragem colaborativa, são reconhecidos os usuários com perfis similares e são recomendados novos objetos de aprendizado a esses usuários.

Em (Ribeiro et al., 2013), os autores utilizam como fonte de dados as hashtags que os alunos acrescentam nos fóruns do ambiente de aprendizagem. Essas hashtags são classificadas e ranqueadas de acordo com o texto das postagens e utilizando a métrica de TF-IDF para geração do vetor de perfil de termos do aluno. Em seguida, materiais são sugeridos a cada aluno de acordo com a similaridade dos vetores de termos dos documentos e do perfil do aluno. Já em (Syed \& Nair, 2017), os autores apresentam um sistema de recomendação de aprendizado personalizada para ser integrado a sistemas de Gerenciamento de Aprendizagem (LMS). A arquitetura proposta visa tornar possível que alunos encontrem material didático relevante, com foco na melhora da experiência de aprendizagem.

Tais abordagens se diferem do presente trabalho em diversos aspectos. Em primeiro lugar, todas necessitam de um cenário previamente estruturado: seja a existência de um repositório corretamente preenchido com o padrão SCORM em (Ferreira \& Raabe, 2010), seja no uso frequente de hashtags em fóruns (Ribeiro et al., 2013) ou mesmo na disponibilidade de um conjunto pré-definido de materiais didáticos (Syed \& Nair, 2017).

A presente proposta utiliza o sistema BAVi, o qual é aplicado para extrair metadados de materiais didáticos automaticamente. Ainda, esta abordagem possui como principal diferencial a recomendação de vídeos. Diferente dos trabalhos citados, esta proposta considera o professor como ator principal do processo de ensino e sua atuação como importante seletor do processo de recomendação. Assim, embora o processo de recomendação se dê de forma automática com base na similaridade entre os recursos didáticos e os conteúdos apresentados no curso, a recomendação se dá inicialmente para o professor e ele escolhe o conteúdo mais adequado ao curso, agindo como facilitador na busca por vídeos em grandes repositórios.

Outras pesquisas relacionadas a recomendação de vídeos para EAD apresentam contribuição para com o presente trabalho. São elas:

- (Basu \& Zimmermann, 2016) apresentam um sistema, chamado videopedia para recomendação de vídeos em blogs. O sistema utiliza o mapeamento dos vídeos através de modelagem por tópicos, ou seja, análise semântica para buscar similaridade entre o vídeo e o texto do blog, fato que também é feito no presente trabalho, ao realizar a análise semântica do texto descritivo da seção do Moodle para complementar a busca por vídeos realizada pelo professor.

- (Deldjoo et al., 2016) propuseram uma solução unificada de recomendação de vídeo do YouTube por meio de colaboração entre redes. As informações auxiliares dos usuários no Twitter são exploradas para solucionar os problemas típicos da recomendação. Este trabalho foi importante para que se investigasse como os discentes poderiam colaborar, no âmbito do Moodle, no sistema de recomendação OVR.

\section{Repositórios de vídeo}

O uso de vídeos educacionais está associado ao armazenamento desse tipo de mídia no próprio servidor do ambiente virtual de aprendizagem, em repositórios de objetos educacionais ou em repositórios específicos para vídeos. No primeiro caso, fatores como catalogação, padronização de metadados, grande demanda por espaço em disco e banda de internet, além de mecanismos adequados para adaptação de entrega de conteúdo, são desafios que tornam essa opção muito custosa. Já os repositórios de objetos educacionais, como o projeto MERLOT (Cafolla, 2006), normalmente apresentam uma boa organização dos conteúdos e dos metadados, mas ainda são 
falhos quando o assunto é adaptação da entrega da mídia (resoluções adaptativas, servidor de streamings etc.) e ainda apresentam desafios de qualidade e reusabilidade dos objetos de aprendizagem armazenados (Silva \& Souza, 2017). Na terceira opção, os repositórios de vídeo de uso geral, como o YouTube, apresentam uma forte capacidade de adaptação na entrega da mídia, mas falham na padronização dos metadados, principalmente quando o vídeo é educacional.

Alguns repositórios, como o VideoAula@RNP, o VideoLectures.NET, Khan Academy e o NTNU, buscam soluções intermediárias, contemplando tanto a parte de metadados, quanto a parte de entrega adaptativa de conteúdo. Apesar de serem idealmente mais completos, os mesmos não possuem a mesma atratividade dos repositórios de uso geral, dado o maior alcance e a simplicidade de se disponibilizar um vídeo nos mesmos, ou seja, a não necessidade de especificar diversos metadados, tão importantes para serviços de recomendação e demais ações relativas a objetos de aprendizagem em geral (Cechinel \& Sicilia, 2012; Ochoa \& Duval, 2009).

Quando o assunto é vídeo educacional, um bom repositório atende às demandas dos usuários e considera fatores que colaboram com o uso efetivo com a intenção de uso do mesmo. Os principais fatores envolvem o nível de experiência do usuário, a plataforma utilizada, o estilo de aprendizagem e a duração do vídeo (Giannakos \& Krogstie, 2016).

A Tabela 1 apresenta os principais repositórios de mídia que contemplam a mídia vídeo, para o Moodle. Vale destacar que foram contemplados somente os plugins disponíveis no site de plugins do Moodle. Apesar de a maioria dos plugins disponibilizarem a entrega adaptativa da mídia, a maior parte não apresenta aspectos que viabilizem características mais voltadas para a aplicação educacional em si, ou seja, metadados, materiais complementares e agrupamento dos vídeos por qualquer que seja o critério.

Tabela 1: Plugins de Repositórios de vídeos para o Moodle.

\begin{tabular}{|c|c|c|c|c|c|c|}
\hline & Moodle & $\begin{array}{c}\text { Personal } \\
\text { YouTube }\end{array}$ & $\begin{array}{c}\text { Ensemble } \\
\text { Video }\end{array}$ & $\begin{array}{c}\text { Planet } \\
\text { eStream }\end{array}$ & $\begin{array}{c}\text { Media } \\
\text { Capture }\end{array}$ & $\begin{array}{c}\text { MEDIAL } \\
\text { Video }\end{array}$ \\
\hline Descrição & $\mathrm{X}$ & $\mathrm{X}$ & $\mathrm{X}$ & $\mathrm{X}$ & $\mathrm{X}$ & $\mathrm{X}$ \\
\hline Streaming adaptativo & $\mathrm{X}$ & $\mathrm{X}$ & & $\mathrm{X}$ & $\mathrm{X}$ & $\mathrm{X}$ \\
\hline Palavras-chave & $\mathrm{X}$ & $\mathrm{X}$ & $\mathrm{X}$ & $\mathrm{X}$ & $\mathrm{X}$ & \\
\hline Agrupamento & & & $\mathrm{X}$ & & & \\
\hline Outros Materiais & & & & $\mathrm{X}$ & & \\
\hline
\end{tabular}

O plugin aqui apresentado oferece a capacidade de criação de descrições, streaming adaptativo e palavras-chave, fazendo uso de um repositório especializado em vídeos educacionais (VideoAula@RNP) e de um serviço de transcrição e tratamento prévio dos vídeos (BAVi).

\section{Sistema BAVi}

Uma descrição completa do funcionamento do sistema BAVi é apresentada no trabalho de Dias et al. (2017), com destaque para o principal objetivo do sistema, ampliar as possibilidades do usuário em encontrar recursos didáticos, a partir do aumento da quantidade de termos associados ao material na relação do mesmo com outros conteúdos existentes em determinado repositório. O enriquecimento e a relação com outros conteúdos é feita com base no sistema de categorização da DBpedia. Em linhas gerais, o fluxo principal pode ser representado pela Figura 1, a qual contempla os seguintes passos do processo de enriquecimento semântico de vídeos:

- Transcrição: Boa parte da informação contida em vídeos educacionais se encontra no áudio (fala do orador). Com base neste fato, técnicas de reconhecimento automático de fala 
são utilizadas para extrair essa informação, gerando um texto transcrito de forma automática. Tal processo é influenciado por variáveis como: a presença de um ou mais falante, música de fundo e palavras ou fonemas desconhecidos ao longo do áudio.

- Anotação Semântica: Associa tags (termos) ao vídeo, sendo o modelo utilizado para anotação, o modelo de tópicos eTVSM (enhanced topic-based vector space model) (Raimond \& Lowis, 2012). Sendo que o modelo foi especializado em associar apenas identificadores de recursos que representam conceitos ao invés de outras classes de palavras.

- Relacionamentos: Gera o relacionamento entre os vídeos do repositório. Com base nas tags semânticas de cada vídeo, é possível comparar o vídeo com o restante do repositório, visando a identificar vídeos com conteúdos similares.

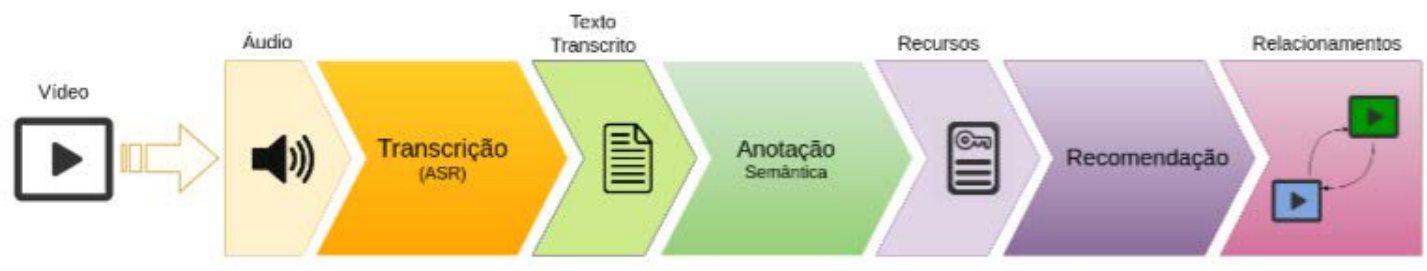

Figura 1: Fluxo Principal do sistema BAVi.

O algoritmo utilizado para recomendar os vídeos é descrito em detalhes por Dias et al. (2017), mas sucintamente o fluxo básico está descrito no Código 1, indicando que cada recurso (termo de busca) relacionado ao vídeo está relacionado à base de conhecimento da DBPedia (categorias). Após associar todos os recursos do vídeo com suas respectivas categorias, os mesmos são relacionados (similaridade), conforme as categorias que possuem seus termos de busca em comum.

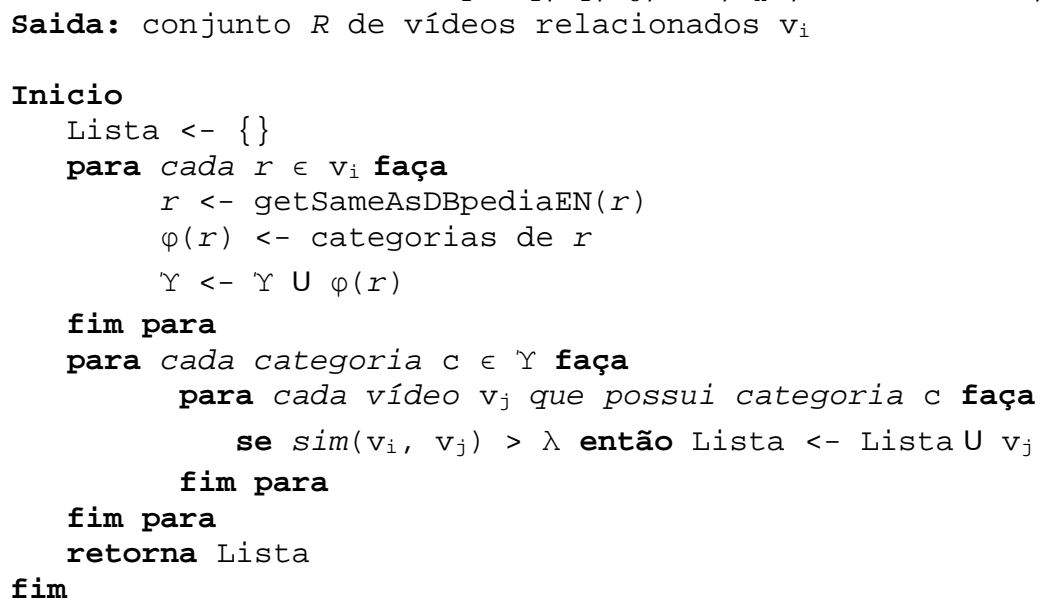

Código 1: Algoritmo para cálculo de similaridade dos videos (Dias et al., 2017).

\section{Sistema de Recomendação Proposto}

Baseado no uso do sistema BAVi para o enriquecimento semântico das palavras-chave de um vídeo e o relacionamento automático desse vídeo com outros armazenados no mesmo 
repositório, foi desenvolvido um sistema de recomendação, conectado ao Moodle na forma de um plugin nomeado OVR (Open Video Recommendation), com as seguintes funcionalidades:

- Busca por vídeo: O professor pode realizar a busca por palavras-chave, de vídeos relacionados a alguma temática. Essa busca pode ser restringida através de um critério de aceitação dos vídeos (notas de um a cinco), solicitando, por exemplo, somente aqueles com notas acima de três.

- Recomendação com base em um vídeo principal: Com base em um vídeo previamente indicado pelo professor, o sistema solicita ao BAVi os vídeos relacionados.

- Recomendação com base no texto do tópico/semana: Com base no texto principal colocado no sumário da semana/tópico, o sistema solicita ao BAVi que execute o processo de anotação semântica desse texto e busque, posteriormente por vídeos relacionados aos termos semânticos encontrados.

Para os três cenários de uso acima descritos, o sistema faz uso das tags semânticas associadasa cada vídeo e das relações de correlação de conteúdos principais entre eles, também pré-processados pela solução. De posse desses dados, o plugin processa a informação para relacionar com os resultados do BAVi. A busca por palavra-chave do professor é comparada com os rótulos dos recursos dos vídeos processados e a busca por materiais relacionados à temática do vídeo selecionado pelo professor é feito pelo plugin através do percorrimento no grafo de relações entre vídeos. Por fim, para a recomendação com base no tópico/semana do curso no Moodle, é utilizada a abordagem de entity linking (Dias et al., 2017), implementada pelo DBpedia Spotlight ${ }^{5}$, para processar o conteúdo textual da descrição da semana/tópico e encontrar os principais conceitos que descrevem os assuntos presentes neste texto. Nesse processo, o sistema de entity linking foi treinado com um subconjunto de recursos da DBpedia, que exclui entidades com rótulos contendo apenas datas, numerais, símbolos, além de instâncias de conceitos, o que removeu do treinamento recursos como nomes de filmes etc. Dessa forma, aumenta-se a probabilidade de serem associados recursos de interesse ao texto. Como resultado do processo, é retornado um conjunto de recursos (conceitos) da DBpedia, os quais representam os principais assuntos do texto analisado.

Após todo esse processamento, o plugin submete o conjunto de recursos para serem comparados com os recursos associados a cada vídeo pelo BAVi. O plugin foi desenvolvido para funcionar seguindo o fluxo padrão de uso descrito abaixo:

1. O Professor utiliza o menu lateral para escolher uma semana/tópico/subseção.

2. O Professor escolhe uma das três formas de recomendar vídeos: busca por palavra-chave, vídeos relacionados a um vídeo de referência ou vídeos relacionados a um texto (sumário). A recomendação pode ainda ser filtrada pela nota mínima atribuída aos vídeos préselecionados pelo OVR.

3. O plugin realiza a requisição de busca no sistema OVR e retorna os resultados.

4. O Professor seleciona os vídeos que lhe interessam e pode ainda mudar o nome que será associado a cada vídeo.

${ }^{5}$ DBpedia Spotlight: https://www.dbpedia-spotlight.org/ 
5. O plugin cria uma atividade do tipo page no Moodle, inserindo nesse objeto os vídeos selecionados pelo professor.

6. Os alunos acessam o objeto page, podendo, além de assistir aos vídeos, atribuir notas aos mesmos.

A Figura 2 apresenta um exemplo do uso do plugin, no qual o professor, após selecionar qual semana/tópico o sistema deve associar a um conteúdo, realiza uma busca por uma palavrachave no plugin. A pesquisa no BAVi é realizada, via web service, e retorna uma tupla com a URL e nome dos vídeos que possuem a referida palavra-chave.

Como nota, vale ressaltar também que os vídeos previamente utilizados em outras atividades são reconhecidos pelo plugin e quaisquer avaliações existentes dos mesmos, feitas por usuários ligados a essas atividades anteriores, são apresentadas ao professor. Isso permite que alunos, de forma indireta, participem também do processo de filtragem, dando ao professor um recurso baseado na opinião dos que irão estudar pelo material escolhido para a seleção de conteúdo e com isso, em teoria, o professor criará uma atividade que será mais bem recebida e possuirá melhor aproveitamento por seus alunos.

videoaulas

\section{Pesquisa:}

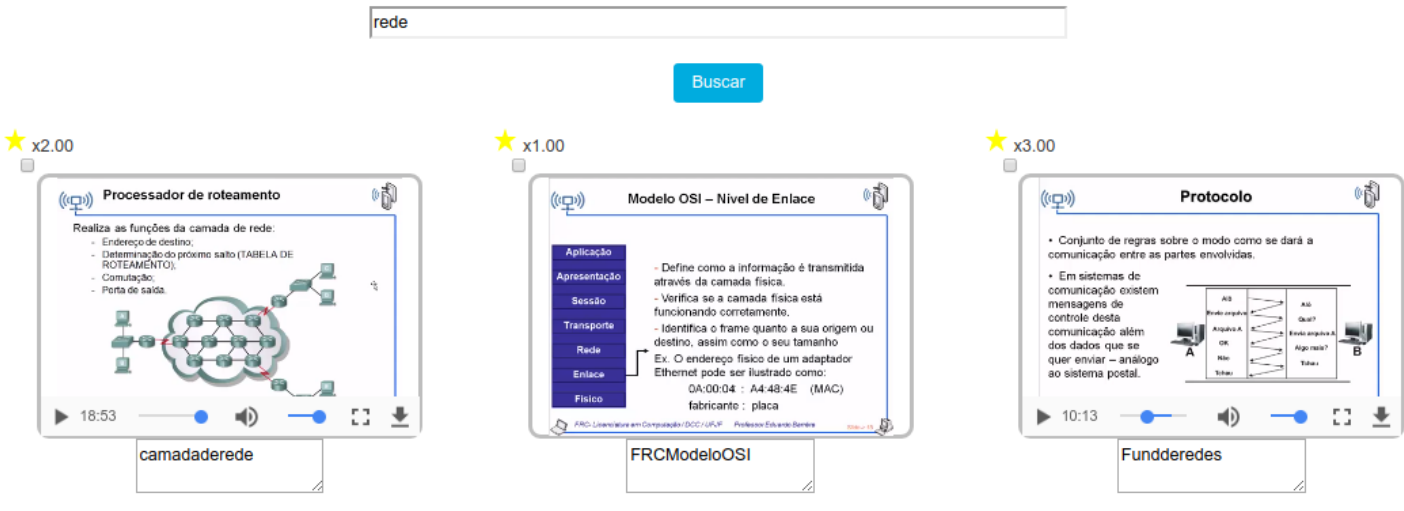

Figura 2: Recomendação por busca de palavra-chave.

Suponha que o professor tenha solicitado ao sistema a recomendação automática de vídeos relacionados ao tema Camada de rede TCP/IP, e que o professor já tenha escolhido, dentre os vídeos retornados pelo plugin, os que devem ser apresentados aos alunos. Neste caso, a Figura 3 apresenta parte do objeto page do Moodle, gerado após o professor concluir o processo de seleção de vídeos recomendados. Vale destacar que com o objeto page gerado, assim como outros objetos do Moodle, é possível atribuir ações de gamificação e controle de acesso, tornando o resultado gerado pelo plugin totalmente compatível com a plataforma em questão. 


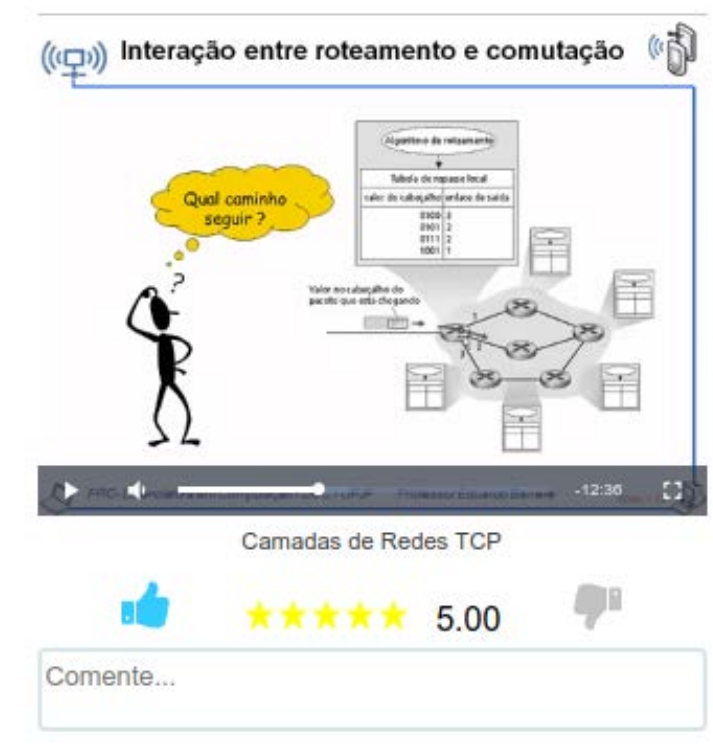

Figura 3: Recomendação por busca de palavra-chave.

O primeiro elemento da Figura 3 é o vídeo recomendado, seguindo pelo título do mesmo abaixo. Após o título temos acesso aos elementos de avaliação dos usuários. As estrelas e os botões de "like" e "dislike" permitem que usuários do Moodle que façam essa atividade deem sua avaliação do conteúdo, enquanto que a caixa de texto no canto inferior da imagem é usada para que os mesmos escrevam um pequeno parágrafo explanando alguma opinião sobre o vídeo.

\section{Estruturação e Explicação dos Principais Componentes do OVR}

O OVR foi desenvolvido na forma de um Bloco do Moodle. Blocos são plugins que adicionam novas funcionalidades à plataforma na forma de ferramentas visuais, presentes, geralmente, nas laterais das páginas dos cursos que as usam. A escolha em desenvolver o OVR com essa abordagem ocorreu pela necessidade de:

- Permitir aos docentes a rápida criação de diversas atividades compostas por diferentes vídeos: Esse raciocínio se dá pelo fato de blocos permitirem um acesso mais rápido a suas funcionalidades, em relação ao tipo de plugin Atividades, também utilizado para a criação de conteúdo a ser usado pelo discente. Em fato, o OVR faz uso de atividades já existentes no Moodle para gerar o seu conteúdo, fato que nos leva ao próximo ponto.

- Evitar a criação de plugins com funcionalidades redundantes no Moodle: A exibição de mídia é contemplada no Moodle na forma de diversas atividades já presentes na instalação do sistema, com plugins dando suporte a exibição de vídeos via HTML5 (suporte nativo dos próprios navegadores web), imagens de todas as extensões comumente usadas e texto (permitindo a inserção de edições no mesmo, como letras em negrito, itálico, sublinhado, diferenciação de tamanho etc.).

- Facilidade de criação de componentes customizados: Blocos oferecem uma possibilidade de criação de funcionalidades muito maior do que outras opções disponíveis, como atividades, dando a opção da criação de páginas como a de busca e seleção de vídeos presentes no plugin.

\subsection{Página de Busca e Seleção de Vídeos}


A página de busca e seleção de vídeos (index.php) faz uso de Asynchronous JavaScript And $X M L$ (AJAX), comunicando-se diretamente com uma API, responsável por requisitar a busca nos servidores do sistema BAVi (Busca Avançada de Vídeo), presente no servidor do Moodle.

A API responde a página de busca, retornando uma lista com todos os vídeos encontrados pelo BAVi (na forma de suas URLs). Tal lista é lida e, através de um processo de inserção de elementos na página via Javascript, tem seus vídeos adicionados, via elementos vídeo do HTML5, para a averiguação e escolha por parte do docente. Vale notar também que para cada vídeo adicionado, uma busca na base local do Moodle é feita para descobrir se o mesmo já fora usado em outra atividade criada pelo OVR e se ele já fora pontuado (via o sistema de estrelas). Caso o vídeo tenha pontuação, a mesma também é exibida para o docente.

Quando um ou mais vídeos são selecionados pelo docente e o mesmo requer a criação da atividade para seus discentes, é usado, novamente, AJAX para requerer ao servidor do Moodle a criação de uma atividade do tipo page com todos os vídeos selecionados.

\subsection{Atividade de Exibição, Pontuação de Vídeos e Exibição e Inserção de Comentários}

Recordando que os vídeos selecionados são exibidos em uma atividade do tipo page para os discentes. Essa atividade permite que elementos de uma página web sejam utilizados para a construção da mesma, ou seja, ela permite que páginas web padrão (HTML + Javascript) sejam criadas e exibidas aos discentes dentro do Moodle.

Essa atividade é utilizada em detrimento da atividade URL (atividade padrão do Moodle que já possui base para a exibição de vídeos), pois a capacidade de se criar uma página web completa permite que façamos uso de Javascript para a criação de novas funcionalidades (neste caso, a criação do sistema de votação/avaliação do vídeo entre um e cinco estrelas, além do acesso a base de dados do Moodle para obter a pontuação já existente de cada vídeo), além de também nos permitir exibir mais de um vídeo por atividade, algo que a atividade URL não dá suporte.

Sempre que o docente gerar uma nova atividade pelo OVR, uma nova page é gerada. São armazenados nas configurações da própria page os dados necessários (scripts e conteúdo HTML com as URLs dos vídeos) para a exibição correta dos vídeos. Como qualquer atividade do Moodle, cada page gerada possui uma entrada única na base de dados do servidor. Vale notar que assim que a mesma é criada, ela é totalmente independente do plugin OVR para a exibição dos vídeos, porém, ela ainda faz uso de arquivos de configuração existentes no plugin para ter acesso a operações mais avançadas (a funcionalidade de classificação do vídeo de um a cinco estrelas requer acesso a arquivos presentes no OVR, caso o mesmo não esteja instalado no servidor, a page ainda exibirá os vídeos normalmente, mas não haverá a opção de classificação do vídeo).

Para acessar a base de dados e obter a classificação de cada vídeo, scripts presentes na page fazem uso de AJAX para acessar a base de dados do servidor. Nesta base existe uma entrada criada pelo OVR para cada vídeo já classificado, assim como sua nota atual (a nota é obtida através de uma simples operação de média aritmética, onde o total de classificações individuais divide o total de estrelas dadas ao vídeo, gerando um valor real de um a cinco). As notas são retornadas em um arquivo, com estrutura semelhante ao comentado na seção 6.1, e são exibidas na forma visual de estrelas para cada vídeo. Cada usuário do Moodle pode realizar um voto com o valor de um a cinco para cada vídeo. Tentativas posteriores ao primeiro voto não são computadas.

Além de classificar um vídeo, usuários do Moodle também podem adicionar comentários escritos aos mesmos. Todos os comentários ligados a um vídeo específico são armazenados 
localmente na base de dados do servidor (e seu acesso pela page também faz uso de scripts com AJAX) e podem ser lidos na atividade de exibição do mesmo.

\section{Experimento}

Além dos Testes de Funcionalidade gerais, foi realizado um experimento com o objetivo de verificar o funcionamento da solução proposta em um cenário real de uso. Para tal, foi escolhida a disciplina de Fundamentos de Redes de Computadores, do curso de Licenciatura em Computação, ofertada na modalidade a distância. Tal escolha ocorreu devido ao fato de os alunos também terem interesse nos aspectos que abordam técnicas e ferramentas para docência, fator que aumentou o envolvimento da turma com o experimento.

Durante a disciplina, o professor comunicou a turma que, em uma determinada semana, iria disponibilizar um conteúdo complementar que faria parte de um experimento e, caso a turma se interessasse em acessar esse conteúdo após assistir aos vídeos, os alunos deveriam responder a um questionário relacionado à forma de recomendação dos vídeos apresentados. De um total de 30 alunos na turma, 15 aceitaram participar do experimento.

Foi utilizada uma base contendo todos os vídeos do serviço VideoAula@RNP (893 vídeos de diversas áreas), complementada com videoaulas sobre redes de computadores disponíveis no YouTube e previamente selecionadas. Esses vídeos foram processados pelo BAVi, gerando a base de busca e relacionamento disponível para o plugin. O professor selecionou vídeos gerais sobre redes de computadores, realizando a busca através da palavra-chave "redes" e escolheu três vídeos para serem disponibilizados aos alunos. Ainda, o professor indicou um vídeo de referência sobre IPV6, o qual gerou uma lista de vídeos relacionados, tendo selecionado dois vídeos para disponibilizar aos alunos.

A dinâmica foi baseada em uma apresentação prévia do projeto, texto informativo, e a disponibilização de dois objetos do tipo page para os alunos, cada um contendo um dos grupos de vídeos selecionados pelo professor. Também foi disponibilizado um questionário, e toda a atividade deveria ser realizada em um prazo de uma semana.

\subsection{Análise dos resultados}

A primeira análise foi em relação aos vídeos retornados ao professor para a seleção dos que seriam disponibilizados para os alunos. Tal fato é de primordial importância para garantir a funcionalidade da ferramenta, ou seja, não apresentar ao professor um grupo de vídeos que estejam desconectados (não relacionados) com o tema pesquisado. Na análise do professor participante da pesquisa, todos os vídeos retornados, em ambos os casos, estavam relacionados com as temáticas de referência, mas nem sempre tinham como tema principal as palavras "redes" ou "IPV6". Já o questionário respondido pelos alunos tem dois focos principais: a percepção dos alunos quanto aos vídeos apresentados e quanto ao uso do plugin em geral.

A Figura 4 apresenta os resultados relacionados aos vídeos recomendados para os discentes. Vale destacar que todos os alunos consideraram que os vídeos tiveram relação com o tema, fato garantido pela seleção prévia do professor. 


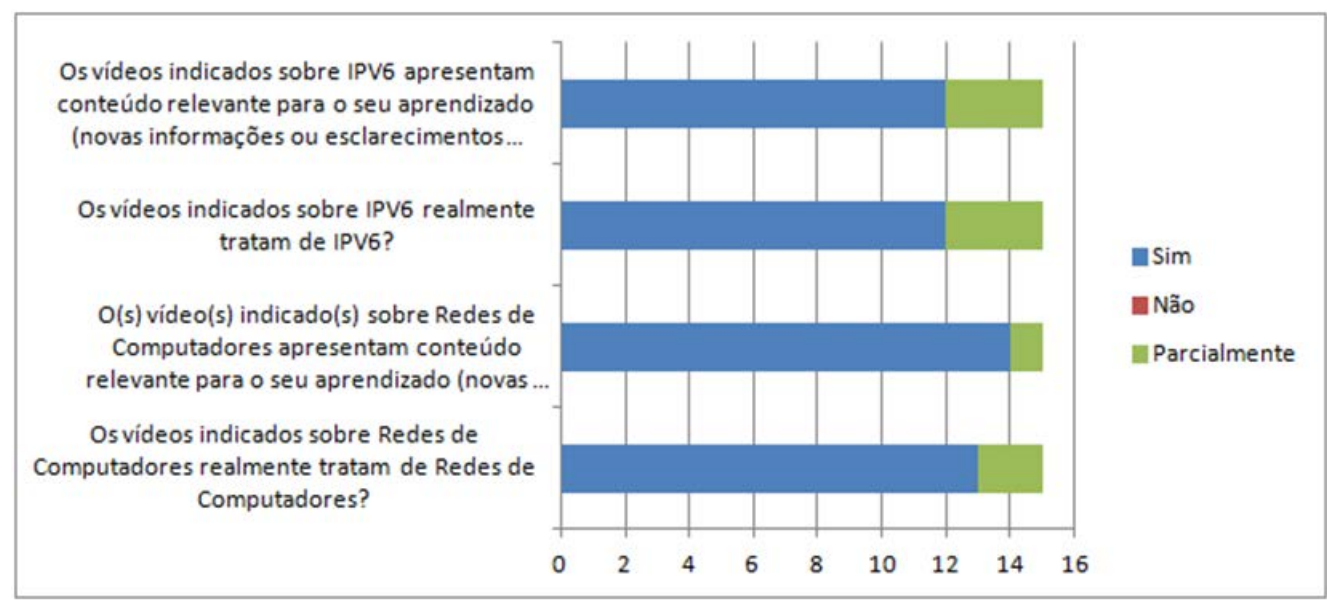

Figura 4: Relação de afinidade entre os vídeos e os temas.

Quanto à relevância dos vídeos, a maioria absoluta dos alunos definiram como satisfatória e os que definiram como parcialmente satisfatória no geral alegaram que já tinham um bom conhecimento sobre o tema. Em complementação a esses dados, o professor foi questionado sobre dois pontos: (1) a pertinência e exatidão desses vídeos em relação às palavras utilizadas para busca e (2) o fato de não ter selecionado todos os vídeos para disponibilização aos discentes. Quanto ao ponto (1), o professor informou que todos os vídeos retornados pelo sistema estavam relacionados, em grau maior ou menor com o tema pesquisa. Já em relação ao ponto (2), informou que alguns vídeos apresentavam conceitos e exemplos muito similares e que selecionou o mais adequado para a turma (relação profundidade do Tema x Curso).

Quando o foco era o plugin, os alunos foram questionados a cerca da recomendação automática de vídeos (previamente aprovados pelo professor) como material complementar a um tópico de uma disciplina, permitindo múltiplas respostas. A Figura 5 apresenta os resultados deste questionamento, com destaque para:

- A maioria dos participantes (55,6\%) considera que a ideia é boa e deveria ser utilizada sempre que possível, conforme a contextualização da dinâmica do curso permita. Tal postura teve como apoio (campo de observações da pesquisa) o fato dos vídeos apresentarem aplicações e outras formas de descreverem o tema.

- Aproximadamente $44,4 \%$ das respostas destacam a preocupação em utilizar esse tipo de recurso tecnológico, tendo como justificativa central (campo de observações da pesquisa) o impacto que vídeos não elaborados pelo professor possam trazer à conduta da disciplina. Tal postura ocorreu, mesmo todos tendo a informação de que o professor é quem faz a escolha final dos vídeos previamente selecionados.

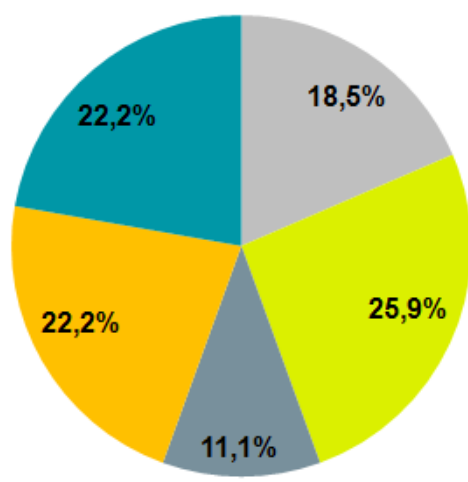

É uma forma interessante de apresentar novos conteúdos ou mesmos novas formas de tratar o tema

Deveria ser utilizada em todos os conteúdos

- Deveria ser utilizado somente em alguns conteúdos

Não deve ser utilizado, pois tira o controle do professor sobre o conteúdo

- Não deve ser utilizado, pois dificulta muito a conduta do professorem relação ao tema. 
Figura 5: Sobre o uso do plugin.

Já em relação à colaboração dos alunos com o repositório de vídeos, através da avaliação dos vídeos assistidos, todos os participantes consideraram relevante tal iniciativa e destacaram também a possibilidade de outras formas de colaboração, como curtir ou não os vídeos e comentários que poderiam ser feitos sobre eles. Todas essas funcionalidades foram incorporadas na versão atual do plugin, vide Figura 3.

A junção dos comentários dos alunos, da avaliação e do quanto os discentes curtiram os vídeos; em conjunto com a ampliação da busca com base em outros metadados do vídeo ou mesmo dos textos existentes na seção do Moodle na qual o OVR será inserido, geram um grande potencial para aprimoramento da recomendação.

\section{Conclusão}

O presente trabalho apresenta um plugin para proporcionar aos professores um sistema de recomendação de videoaulas no ambiente Moodle. As videoaulas são enriquecidas semanticamente pelo sistema BAVi e podem ser oriundas de repositórios dedicados ou distintos, previamente selecionados pelo professor para complementar os conteúdos previstos para a disciplina. Diferente de outras abordagens presentes na literatura, este plugin coloca o professor como ponto central do processo de recomendação. A versão atual do OVR está disponível no portal EduCapes através do link: https://educapes.capes.gov.br/handle/capes/431225.

A recomendação se inicia com a seleção de conteúdo de interesse do professor, o qual recebe automaticamente um conjunto de vídeos à partir de uma busca, do conteúdo presente no tópico da disciplina ou à partir de um vídeo de referência. Após a escolha, o aluno pode assistir ao conteúdo selecionado pelo professor e, a partir desse, navegar por vídeos correlatos através das sugestões na tela. As informações dos vídeos dos repositórios são complementadas através do registro de número de acessos e de colaborações dos alunos, que podem curtir e escrever comentários para cada vídeo. Ao disponibilizar o acesso a uma base de vídeos especializada (subgrupo do repositório ou vários repositórios integrados) é possível organizar vídeos por grupos de características/níveis de conteúdo, baseados em aspectos descritos em padrões, como o SCORM, por exemplo.

O experimento realizado demonstrou que o funcionamento do plugin atende aos seus objetivos e possibilita uma forma rápida de oferecer material complementar aos alunos, permitindo também que os usuários do plugin possam colaborar com as bases de vídeos dos repositórios, através da avaliação dos vídeos, de comentários ou mesmo pelo simples acesso (contagem de acessos).

A indicação de vídeos, através do OVR, que podem conter conteúdos com abordagens pedagógicas diferentes ou mesmo abordando temas não contemplados no material didático básico da disciplina, foi sinalizado pelos participantes da pesquisa, vide Figura 4, como um ponto que diminuiu o controle do professor sobre o andamento da disciplina. Tal situação pode trazer aspetos que necessitem de outras abordagens pedagógicas, como por exemplo, trazendo dinâmicas de sala de aula invertida (Abeysekera \& Dawson, 2015). Esse tipo de cenário foi caracterizado na pesquisa $(44,4 \%=22,2 \%+22,2 \%)$ como um desmotivador para o uso desse tipo de plugin. Sendo assim, conforme o conteúdo e abordagem de cada vídeo, o mesmo pode criar a necessidade de mudança na abordagem do professor e tal situação só pode ser minimizada com base em especificações completas dos vídeos como objetos de aprendizagem, inserindo esses elementos também na fase de busca dos vídeos. As mudanças atuais no plugin, 
likes e/ou comentários dos alunos, permitem a realização de uma pesquisa com foco na forma de interação dos alunos com os vídeos recomendados e a retroalimentação dessas informações no sistema, permitindo que os professores a utilizem como parâmetros em buscas futuras.

Como trabalhos futuros, espera-se utilizar informações do aluno para complementar as recomendações automáticas após a sugestão do professor. Neste contexto, o histórico de notas pode ser utilizado em conjunto com informações do SCORM para selecionar materiais complementares de acordo com o nível de dificuldade esperado para um aluno. Ainda, espera-se permitir que o professor atue no processo de recomendação através de adaptação de parâmetros do plugin, permitindo comportamentos distintos para cada curso. Espera-se também adicionar a capacidade de filtrar automaticamente os resultados de pesquisa pelo sistema de avaliação dos usuários (ordenação automática dos vídeos com base no número de estrelas e porcentagem de likes).

\section{Agradecimentos}

Agradecemos ao financiamento da CAPES, através de recursos provenientes do Edital 03/2015, que viabilizou a execução do projeto. Agradecemos também à RNP pelo financiamento do projeto BAVi, o qual permitiu o desdobramento e criação de outros projetos, incluindo o que resultou no presente trabalho.

\section{Referências}

Abeysekera, L. \& Dawson, P. (2015). Motivation and cognitive load in the flipped classroom: definition, rationale and a call for research. Journal Higher Education Research \& Development , 34 (1), 1-14. doi: 10.1080/07294360.2014.934336

Allen, E., \& Seaman, J. (2017). Digital Learning Compass: Distance Education Enrollment Report $2017 \quad$ (Tech. Rep.). Retrieved from https://onlinelearningsurvey.com/reports/digtiallearningcompassenrollment2017.

Barrére, E., Vitor, M.A. \& Almeida, M. A. (2017). Ampliação das Possibilidades de Gamificação no Moodle. Proceedings of XXVIII Brazilian Symposium on Computers in Education (Simpósio Brasileiro de Informática na Educação - SBIE), 605-614. doi: 10.5753/cbie.sbie.2017.605

Brame, C. J., \& Perez, K. E. (2016). Effective educational videos: Principles and guidelines for maximizing student learning from video content. Proceedings of CBE-Life Sciences Education, 15. doi: 10.1187/cbe.16-03-0125

Brandão, W. C., Neves, D. E., \& Ishitani, L. (2017). Automatic content recommendation and aggregation according to scorm. Informatics in Education - An International Journal, 16(2), 225-256. [GSSearch]

Cafolla, R. (2006). Project merlot: Bringing peer review to web-based educational resources. Journal of Technology and Teacher Education, 14, 313-323. [GSSearch]

Cechinel, S.-A.-S., C., \& Sicilia, M. (2012). Automated quality assessment of learning objects inside repositories. Brazilian Journal of Computers in Education, 20(3), 43-59. doi: 10.5753/rbie.2012.20.03.43. 
Costa, E., Aguiar, J., \& Magalhães, J. (2013). Sistemas de recomendação de recursos educacionais: conceitos, técnicas e aplicações. Proceedings of Jornada de Atualização em Informática na Educação, 1(1). [GSSearch]

Damnjanovic, J.-S., Vesna, \& Mijatovic, I. (2015). Factors affecting the effectiveness and use of moodle: Students' perception. Proceedings of Interactive Learning Environments, 23, 10494820. doi: $10.1080 / 10494820.2013 .789062$

Deldjoo. Y., Elahi, M., Cremonesi, P. Garzotto, F. Piazzolla, P. \& Quadrana, M. (2016). Content-Based Video Recommendation System Based on Stylistic Visual Features. Journal on Data Semantics, 5(2), 99-113. doi: 10.1007/s13740-016-0060-9

Dias, L. L., Barbosa, J. S., Barrére, E. \& Souza, J. F. de (2017). An Approach to Identify Similarity Among Educational Resources Using External Knowledge Bases. Brazilian Journal of Computers in Education, 25(2), 18-37. doi: 10.5753/rbie.2017.25.02.18

Ferreira, V. H., \& Raabe, A. L. A. (2010). LORSys: Um sistema de recomendação de objetos de aprendizagem SCORM. Revista Novas Tecnologias na Educação, 8(2). doi: $\underline{10.22456 / 1679-1916.15223}$

Giannakos, M. N., Jaccheri, L. \& Krogstie, J. (2016). Exploring the relationship between video lecture usage patterns and students attitudes. British Journal of Educational Technology, 47(6), 1259-1275. doi: 10.1111/bjet.12313

Halawa, H. E., Hamed, E. M. R., \& Shehab, M. E. (2015). Personalized e-learning recommendation model based on psychological type and learning style models. Proceedings of 2015 IEEE seventh international conference on intelligent computing and information systems. 578-584. doi: 10.1109/IntelCIS.2015.7397281

Limongelli, C., Lombardi, M., Marani, A., Sciarrone, F. \& Temperini, M. (2016). A recommendation module to help teachers build courses through the moodle learning management system. New Review of Hypermedia and Multimedia, 22, 58-82. doi: $\underline{10.1080 / 13614568.2015 .1077277}$

Nascimento, P. do, Barreto, R., Primo, T., Gusmão, T. \& Oliveira, E. (2017). Recomendação de objetos de aprendizagem baseada em modelos de estilos de aprendizagem: Uma revisão sistemática da literatura. Proceedings of Simpósio brasileiro de informática na educação, 213-222. doi: 10.5753/cbie.sbie.2017.213

Ochoa, X., \& Duval, E. (2009). Quantitative analysis of learning object repositories. IEEE Transactions on Learning Technologies, 2(3), 226-238. doi: 10.1109/TLT.2009.28

Pirhonen, J., \& Rasi, P. (2016). Student-generated instructional videos facilitate learning through positive emotions. Journal of Biological Education, 51(3), 215-227. doi: $\underline{10.1080 / 00219266.2016 .1200647 ~}$

Raimond, Y., \& Lowis, C. (2012). Automated interlinking of speech radio archives. Proceedings of Workshop on Linked Data on the Web, 937. [GSSearch]

Ribeiro, F. A. A., Fonseca, L. C. C., \& Freitas, M. S. (2013). Recomendando objetos de aprendizagem a partir das hashtags postadas no moodle. Proceedings of Simpósio brasileiro de informática na educação, 82-91. doi: 10.5753/cbie.sbie.2013.82

Silva, J. W. da, \& Souza, C. de (2017). Repositórios de objetos de aprendizagem: características; classificações; limitações e tendências. Proceedings of Simpósio brasileiro de informática na educação, 61-70. doi: 10.5753/cbie.sbie.2017.61 
Syed, T. A., Palade, V., Iqbal, R. \& Nair, S. S. K. (2017). A personalized learning recommendation system architecture for learning management system. Proceedings of the 9th international joint conference on knowledge discovery. doi: $10.5220 / 0006513202750282$

Tibaná-Herrera, G., Fernández-Bajón, M. T. \& Moya-Anegón, F. de (2018). Global analysis of the e-learning scientific domain: a declining category? Scientometrics - An International Journal for all Quantitative Aspects of the Science of Science, Communication in Science and Science Policy, 114(2), 675-685. doi: 10.1007/s11192-017-2592-7 